\title{
Análise das relações entre estratégia de produção, práticas e desempenho operacional
}

\author{
Eliciane Maria da Silva ${ }^{a *}$, Fernando César Almada Santos ${ }^{\mathrm{b}}$, \\ Mário de Castro ${ }^{c}$ \\ a"eimsilva@unimep.br, UNIMEP, Brasil \\ balmada@sc.usp.br, USP, Brasil \\ cmcastro@icmc.usp.br, USP, Brasil
}

\section{Resumo}

Este trabalho teve o objetivo de verificar quais são as melhores práticas de produção para o setor moveleiro no Brasil, mediante a análise das relações entre as práticas de produção implantadas e prioridades competitivas e, também, entre indicadores de desempenho. A revisão teórica centrou em definir o pressuposto para o estudo das melhores práticas e realizar uma sistematização da literatura. Posteriormente, foi desenvolvida a pesquisa prática mediante uma survey em 99 empresas. Para a análise dos dados, foi empregada a técnica de modelagem de equações estruturais com o método de estimação dos mínimos quadrados ponderados. Os resultados revelaram que as práticas nas áreas de desenvolvimento de novos produtos, recursos humanos e planejamento e controle de produção estavam alinhadas à estratégia de produção e contribuíram para a melhoria de indicadores de desempenho operacional, sendo assim consideradas melhores práticas para o contexto estudado. Foram feitas discussões e sugestões para pesquisas futuras. Palavras-chave

Melhores práticas. Estratégia de produção. Desempenho operacional. Modelagem de equações estruturais. Setor moveleiro.

\section{Introdução}

Existe um número considerável de estudos na área de gestão de operações que analisam a relação entre práticas e desempenho por meio da perspectiva de melhores práticas de produção. A implementação de melhores práticas vem sendo tratada como relevante, pois pode conduzir para a melhoria de desempenho, e consequentemente, guiar as empresas para um aumento de competitividade (DAVIES; KOCHHAR, 2002).

Estudos tradicionais a respeito de melhores práticas tiveram como modelos práticas japonesas, tais como: Just in time, Kaizen e sistema de troca rápida de ferramentas (SMED - Single Minute Exchange of Die) (WOMACK; JONES; ROOS, 1990). Contudo, a evolução das pesquisas nessa área revelou que a implantação dessas práticas em firmas diferentes pode levar a níveis de desempenhos distintos (NARASIMHAN; SWINK; KIM, 2005).
Davies e Kochhar (2002) afirmam que o conceito de melhores práticas necessita de análises mais aprofundadas, pois as melhores práticas de uma empresa bem-sucedida podem não ser aplicáveis no contexto de outras organizações. Os autores reforçam que as análises dos efeitos das práticas sobre desempenho são ainda superficiais - as pesquisas são realizadas em contextos gerais, envolvendo diferentes setores e tipos de produtos. Outros pesquisadores também confirmam essa limitação, sugerindo uma discussão mais apropriada das melhores práticas para contextos específicos (VOSS, 1995; SILA; EBRAHIMPOUR, 2005; UNGAN, 2005). Ademais, Beaumont (2005) diz que as definições de melhores práticas diferem entre autores e é ainda insuficiente na literatura. Essas lacunas identificadas motivam a realização do presente estudo que centra em responder o seguinte problema de pesquisa: 
- Quais são as melhores práticas de produção, relacionadas à estratégia de produção, que contribuem para a melhoria do desempenho operacional no setor moveleiro no Brasil?

A escolha desse setor se justifica por representar uma parcela não desprezível da economia brasileira. Possui $1,4 \%$ das receitas brutas da indústria de transformação, cujo valor foi de R\$ 17 bilhões em 2005. Representa cerca 3,6\% do total de trabalhos alocados, o equivalente a 227,6 mil empregos (INSTITUTO..., 2006). Este setor se encontrou prejudicado, nos anos de 2007 e 2008, no mercado externo em razão da política econômica instável, o que contribuiu ainda mais para o aumento da competição no mercado doméstico (CENTRO..., 2007). No âmbito prático, acredita-se que o presente estudo possa auxiliar gerentes de produção a entender melhor a relação entre a implantação de práticas e os resultados alcançados.

No ambiente acadêmico, pretende-se esclarecer as lacunas encontradas nessa área de pesquisa.

Portanto, este trabalho tem o objetivo de verificar quais são as melhores práticas de produção para o setor moveleiro mediante a análise das relações entre as práticas implantadas e à estratégia de produção e, também, entre a melhoria de indicadores de desempenho operacional.

A estrutura deste artigo está dividida da seguinte forma. Primeiro, é realizada uma revisão teórica. Posteriormente, são descritas a metodologia da pesquisa e as técnicas de análises dos dados. Na sequência, são apresentadas as discussões dos resultados. Por fim, são feitas as considerações finais, descrevendo limitações da pesquisa e sugestões para trabalhos futuros.

\subsection{Revisão teórica e hipóteses de pesquisa}

Dois elementos centrais definem a estratégia de produção como uma subestratégia funcional: prioridades competitivas e decisões de investimentos em áreas estruturais e infraestruturais de produção (SKINNER, 1969; MILLER; ROTH, 1994; HAYES et al., 2004).

As prioridades competitivas são um dos elementoschave da estratégia de produção e indicam uma declaração de quais funções a manufatura deve executar com êxito. Ou seja, como as atividades de produção devem ser definidas em termos de capacitações para as empresas competirem com sua estratégia de negócio (MILLER; ROTH, 1994). Skinner (1969, 1978) apresentou uma definição semelhante introduzindo o termo missão da manufatura, estabelecendo quais operações devem ser realizadas para o sucesso do negócio. Normalmente, essas operações ou habilidades incluem os conceitos de qualidade, custo/eficiência, entrega/responsabilidade e flexibilidade (MILLER; ROTH, 1994). Essas quatro prioridades competitivas foram as de maior inserção entre os estudos pesquisados (SKINNER, 1969; SCHMENNER, 1981; WHEELWRIGHT, 1984; GERWIN, 1987; HÖRTE; LINDBERG; TUNÄLV, 1987; SWAMIDASS; NEWELL, 1987; ADAM; SWAMIDASS, 1989; LEONG; SNYDER; WARD, 1990; GARVIN, 1993; HILL, 1993; SCHROEDER, 1993; STONENRAKER; LEONG, 1994; PIRES, 1995; VACHON; KLASSEN, 2006; SILVA et al., 2008). Assim, tais prioridades foram adotadas no presente trabalho.

0 segundo elemento central da estratégia de produção se refere às decisões de investimentos em áreas estruturais e infraestruturais (HAYES; WHEELWRIGHT, 1984; FINE; HAX, 1985). As áreas estruturais são aquelas de investimentos relacionados a capacidade, instalações industriais, tecnologia e integração vertical. Já as áreas infraestruturais se classificam em adoção de novas políticas ou programas de ação que envolvam as áreas de recursos humanos, gerência da qualidade, planejamento e controle da produção e arranjo físico (HAYES; WHEELWRIGHT, 1984) como também, relacionamento com os fornecedores, desenvolvimentos de novos produtos (PAIVA; CARVALHO JUNIOR; FENSTERSEIFER, 2004) e gestão ambiental (ANGELL; KLASSEN, 1999; SILVA et al., 2008).

Investimentos em práticas bem-sucedidas como Just-in-Time (JIT) e Total Quality Management (TQM) também são discutidos com ênfase estratégica nas unidades de negócio (ADAM; SWAMIDASS, 1989), motivando estudos na investigação do conceito de melhores práticas de produção.

São encontrados pelo menos dois pressupostos para o estudo das melhores práticas de produção: a) melhores práticas são aquelas adotadas por empresas de manufatura de classe mundial. Essas empresas adotam práticas que são reconhecidas no âmbito internacional com a finalidade de alcançar performance igual ou superior à de companhias que competem globalmente (VOSS, 1995; FLYNN et al., 1997; CAMP, 1999; LAUGEN; BOER; FRICK, 2005). Observa-se que nessa abordagem as melhores práticas conduzem para alta performance ou desempenho superior das firmas; e b) melhores práticas são aquelas relacionadas com a estratégia de produção e que proveem melhoria de desempenho (MILLS; PLATTS; GREGORY, 1995; BEAMON, 1999; KETOKIVl; SCHROEDER, 2004). Nessa definição, as melhores práticas não são vistas como universais, pois práticas são as melhores dependendo do contexto das organizações. Para Ugan (2005), o conceito de melhores práticas é muito subjetivo, visto que as melhores práticas para uma empresa podem não ser as melhores para outras. 
Pesquisadores dessa abordagem baseiam-se na teoria contingencial, cujo precursor foi Skinner (1969) que enfatizou a adequação entre sistemas de produção e prioridades competitivas. A teoria contingencial sustenta que organizações adaptam suas estruturas para se adequar às mudanças de fatores contextuais. As contribuições teóricas e práticas desta abordagem são: a) a identificação de variáveis contingenciais importantes que distinguem entre contextos; b) o agrupamento de contextos diferentes baseados nas variáveis contingenciais; e c) a determinação de projetos internos mais efetivos da organização ou de grupos majoritários (SOUSA; VOSS, 2008).

Ketokivi e Schroeder (2004) concluíram que algumas práticas certamente são melhores que outras quando estão dirigidas para as prioridades estratégicas. Práticas de produção examinadas no referido estudo influenciaram o desempenho operacional se elas estivessem implementadas por essa razão. Beaumont (2005) e Davies e Kochhar (2002) também afirmaram que na manufatura as melhores práticas adotadas deveriam ser implantadas para apoiar a estratégia e para ter impacto positivo sobre as medidas de performance. Ademais, os autores reforçam ser importante a análise dos efeitos adversos de outros indicadores de desempenho e, também, a análise da necessidade de infraestrutura de programas de ação que são essenciais para apoiar as melhores práticas a serem adotadas. De acordo com a base teórica investigada até o momento, a primeira hipótese do presente estudo afirma que:

- H1: Prioridades competitivas afetam positivamente as práticas de produção.

Observou-se também ser imprescindível a análise dos indicadores de desempenho nos estudos de melhores práticas. A saber, um indicador de desempenho é conceituado como uma variável que mede a eficácia ou eficiência parcial ou total de um processo, de acordo com o objetivo ou planejamento proposto (FORTUIN, 1988). Nos estudos investigados (por exemplo, YOUNDT et al., 1996; BOYER, 1998; GILLEY; RASHEED, 2000; KOTHA; SWAMIDASS, 2000; DÍAZ; GIL; MACHUCA, 2005; FYNES; VOSS; BÚRCA, 2005; SILA; EBRAHIMPOUR, 2005; PUJARI,
2006; SILA, 2007) foi encontrada a relação de práticas de produção com pelo menos três construtos de indicadores de desempenho: a) indicadores de desempenho de áreas específicas de produção, b) indicadores de desempenho gerais de produção e c) indicadores de desempenho de negócio.

Portanto, a segunda hipótese de pesquisa para o presente trabalho afirma que:

- H2: Práticas de produção afetam positivamente o desempenho operacional.

A Figura 1 ilustra o modelo conceitual do presente estudo. Mediante o modelo conceitual, é analisada a relação entre a estratégia de produção (prioridades competitivas) e as práticas (ou programas de ação) adotadas (os) em áreas estratégicas consideradas pelas empresas (H1). Também, é analisada a relação entre tais práticas e a melhoria do desempenho operacional (H2). 0 desempenho operacional é representado por medidas gerais de produção e, também, medidas específicas, conforme as áreas em que foram realizados os investimentos em práticas.

Observa-se que a análise dessas duas hipóteses ( $\mathrm{H} 1$ e H2) satisfaz o pressuposto do conceito de melhores práticas encontrado em: Mills, Platts e Gregory (1995), Ketokivi e Schroeder (2004), Beaumont (2005), Davies e Kochhar (2002), Ungan (2005) e Schroeder et al. (2002). Em outras palavras, as melhores práticas serão aquelas que estarão positivamente relacionadas com a estratégia de produção $(\mathrm{H} 1)$ e que, também, influenciarão positivamente o desempenho operacional (H2). A próxima seção aborda a metodologia utilizada na seleção das empresas, coleta de dados e as técnicas usadas na análise dos dados.

\section{Metodologia}

0 método da pesquisa de campo foi uma survey definida como exploratória (PINSONNEAULT; KRAEMER, 1993; FOWLER, 2002; SOUSA; VOSS, 2008) e de corte transversal (FREITAS et al., 2000). Segundo Pinsonneault e Kraemer (1993), a pesquisa survey exploratória busca maior familiaridade com um determinado tópico para se obter conceitos preliminares. Neste contexto, é usada para descobrir

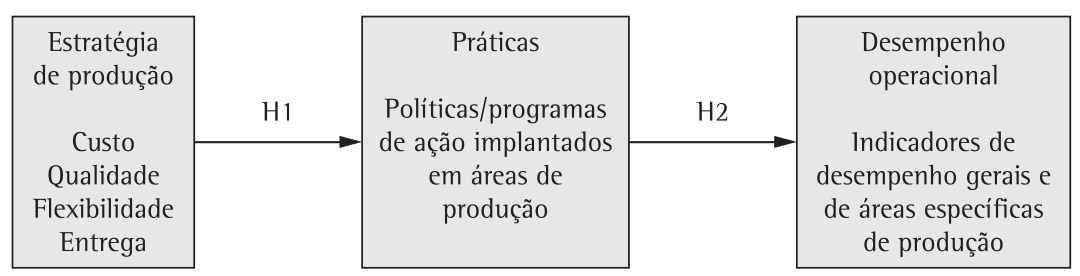

Figura 1. Modelo conceitual. 
mudanças que ocorrem em populações de interesses, refinando a avaliação dos conceitos. 0 pesquisador centraliza em quais conceitos devem ser medidos e como mensurá-los. A survey exploratória também é usada para descobrir e aumentar novas possibilidades e dimensões da população de interesse. Assim, o presente estudo primeiramente buscou saber quais eram as variáveis que representavam os conceitos de prioridades competitivas, práticas de produção e de desempenho usadas pelo setor moveleiro. Posteriormente tais conceitos foram analisados e relacionados por métodos estatísticos apresentados na Seção 3 deste trabalho. A pesquisa survey também foi definida como sendo de corte transversal (FREITAS et al., 2000), uma vez que a coleta de dados em cada empresa ocorreu em um só momento.

As variáveis do questionário foram primeiramente fundamentadas em estudos prévios (por exemplo, VICKERY; DROGE; MARKLAND, 1997; WARD; MCCREERY; RITZMAN, 1998; CUA; McKONE; SCHROEDER, 2001), cujas escalas foram do tipo Likert de 1-5, 1-6 ou 1-7 níveis. Abordagens similares com escalas variadas foram também encontradas em outros estudos (Ll et al., 2006; SWINK; NARASIMHAN; WANG, 2007).

Foram realizados dois testes piloto. No primeiro teste, seis acadêmicos, dois desses com experiência no setor pesquisado, foram convidados para participar com o objetivo de aperfeiçoar a coerência das questões e analisar a extensão do questionário. 0 segundo teste piloto foi aplicado a nove gerentes de produção in loco em fábricas do setor moveleiro, localizadas em Bento Gonçalves (RS), com o objetivo de tornar as variáveis mais específicas para o setor. Então, algumas questões foram modificadas ou descartadas, tornando o questionário mais objetivo e fortalecendo o conteúdo.

A seleção das empresas ocorreu por meio de quatro listagens de associados dos polos moveleiros de São Bento do Sul (SC), Rio Negrinho (SC), Mirassol (SP) e Bento Gonçalves (RS). No total havia 564 empresas associadas. Todas foram contatadas via telefone para participarem da pesquisa, respondendo ao questionário pela internet ou por intermédio de entrevista pessoal. Doze optaram por responder ao questionário eletronicamente e 87 foram visitadas in loco, totalizando 99 firmas pesquisadas, o equivalente a $17,55 \%$ de taxa de resposta. Essa taxa mostra-se similar àquelas observadas em outras pesquisas da área de administração da produção, conforme sistematizado por Synodinos (2003).

0 teste de Kruskal-Wallis foi aplicado para cada variável do questionário a fim de checar se houve diferença significativa em nível de significância 0,05 entre os dois grupos de respondentes (Internet e entrevistas in loco). Níveis descritivos ( $p$-value) oscilaram de 0,99 a 0,25. Portanto, não houve diferença significativa entre os dois grupos de respondentes. Os entrevistados foram diretores ou gerentes de produção. Juntamente com as entrevistas in loco por meio do questionário estruturado, foram realizadas, também, observações diretas no chão de fábrica. A pesquisa de campo iniciou-se na segunda quinzena de novembro de 2006 e encerrou-se no final de julho de 2007.

0 Quadro 1 apresenta características gerais das empresas pesquisadas. Observa-se que a maior parcela das firmas (59\%) possuía entre 100 e 499 empregados, consideradas, portanto, de médio porte pelo critério de número de funcionários estabelecido pelo Sebrae (2008).

As empresas manufaturavam uma variedade alta de linhas de produtos para móveis residenciais (dormitórios, armários e estantes, cozinhas, entre outros) e de escritório, cujo sistema produtivo em função do fluxo de produto, se classificava por lotes ou intermitente (prevalecendo a programação sob encomenda - 66\%). Além disso, o período de programação da produção era predominantemente estabelecido em até uma semana (71\%). As vendas ocorriam em todo território nacional e, também, em menor quantidade no mercado internacional (Quadro 1).

\section{Análise dos dados}

Para analisar a relação entre os construtos das hipóteses $\mathrm{H} 1$ e $\mathrm{H} 2$, foi empregado o método de modelagem de equações estruturais (Structural Equation Modeling, SEM), usando o sistema SAS, por meio do procedimento CALIS (HATCHER, 1994).

Inicialmente a unidimensionalidade dos construtos foi avaliada pela técnica de Análise Fatorial Exploratória (AFE) com análise de componentes principais e rotação varimax. A avaliação dos critérios da raiz latente (autovalor) e do teste scree selecionaram treze fatores latentes. As análises da medida de adequação amostral (Measure of Sampling Adequacy-MSA), das cargas fatoriais e das comunalidades de cada variável conduziram à exclusão de algumas variáveis, cujos valores não foram satisfatórios. Os treze construtos latentes identificados foram nomeados de: a) Prioridade Competitiva de Flexibilidade (PCF); b) Práticas de Tecnologia para Móveis de Painéis (PTECP); c) Práticas de Desenvolvimento de Novos Produtos e de Mix (PDNPM); d) Práticas de Planejamento e Controle de Produção (PPCP), e) Práticas de Recursos Humanos Tradicionais (PRHT); f) Práticas de Recursos Humanos Avançadas (PRHA); g) Desempenho de Volume de Inventário (DVI); h) Desempenho de Planejamento 
Quadro 1. Caracterização geral das empresas pesquisadas.

\begin{tabular}{|c|c|c|c|}
\hline Variáveis & \multicolumn{2}{|c|}{ Escalas } & $\begin{array}{l}\text { Percentual } \\
\quad(\%)\end{array}$ \\
\hline \multirow{3}{*}{$\begin{array}{l}\text { Dimensão da } \\
\text { área fabril }\end{array}$} & \multicolumn{2}{|c|}{1.000 até $20.000\left(\mathrm{~m}^{2}\right)$} & 84 \\
\hline & \multicolumn{2}{|c|}{ Superior a 20.000 a $40.000\left(\mathrm{~m}^{2}\right)$} & 9 \\
\hline & \multicolumn{2}{|c|}{ Superior a $40.000\left(\mathrm{~m}^{2}\right)$} & 7 \\
\hline \multirow{3}{*}{$\begin{array}{l}\text { Número de } \\
\text { funcionários }\end{array}$} & \multicolumn{2}{|c|}{50 a 99} & 35 \\
\hline & \multicolumn{2}{|c|}{100 a 499} & 59 \\
\hline & \multicolumn{2}{|c|}{ Superior a 500} & 6 \\
\hline \multirow{8}{*}{$\begin{array}{l}\text { Principal } \\
\text { matéria-prima }\end{array}$} & \multicolumn{2}{|c|}{$\begin{array}{l}\text { MDF cru, aglomerado, } \\
\text { fita de bordo, MDF revestido }\end{array}$} & 33 \\
\hline & \multicolumn{2}{|c|}{$\begin{array}{l}\text { MDF cru, Fita de Bordo, MDF } \\
\text { revestido, revestimento melamínico }\end{array}$} & 7 \\
\hline & \multicolumn{2}{|c|}{ MDF cru e MDF revestido } & 15 \\
\hline & \multicolumn{2}{|c|}{ Pínus } & 24 \\
\hline & \multicolumn{2}{|c|}{ Outras madeiras maciças e pínus } & 9 \\
\hline & \multicolumn{2}{|c|}{ Outras madeiras maciças e MDF } & 9 \\
\hline & \multicolumn{2}{|c|}{ Tecido e espuma } & 2 \\
\hline & \multicolumn{2}{|c|}{ Aglomerado, MDF, aço } & 1 \\
\hline \multirow{3}{*}{$\begin{array}{l}\text { ldade da empresa } \\
\text { (conforme ano } \\
\text { de fundação) }\end{array}$} & \multicolumn{2}{|c|}{ Até 9 anos } & 12 \\
\hline & \multicolumn{2}{|c|}{ Acima de 9 a 39 anos } & 63 \\
\hline & \multicolumn{2}{|c|}{ Acima de 40 anos } & 25 \\
\hline & \multicolumn{2}{|c|}{ Armário e estantes/ infantis/escritório } & 1 \\
\hline & $\begin{array}{r}\text { Dormitórios/arm } \\
\text { escrit }\end{array}$ & $\begin{array}{l}\text { ários e estantes/ } \\
\text { ório }\end{array}$ & 10 \\
\hline Principais & $\begin{array}{r}\text { Dormitórios/arm } \\
\text { escritório/infa } \\
\text { lavan }\end{array}$ & $\begin{array}{l}\text { ários/ estantes/ } \\
\text { tis/cozinhas/ } \\
\text { deria } \\
\end{array}$ & 35 \\
\hline produtos & Mesas e & assentos & 1 \\
\hline ou linha de & Escrit & ório & 6 \\
\hline & Dormitórios/arm & ários e estantes & 31 \\
\hline & Assentos ( & stofados) & 8 \\
\hline & $\operatorname{lnfa}$ & & 2 \\
\hline & Mesa/a & ssento & 3 \\
\hline & Dormi & tórios & 3 \\
\hline Classificação & Retil & neo & 61 \\
\hline do produto & Retilíneo e & torneado & 39 \\
\hline & & $\begin{array}{c}\text { Em série/ } \\
\text { por lotes } \\
\text { (intermitente) }\end{array}$ & 100 \\
\hline & produção & Por projeto & - \\
\hline & & $\begin{array}{l}\text { Por família de } \\
\text { produto (célula) }\end{array}$ & - \\
\hline & Proqramcõ & Por estoque & 34 \\
\hline Sistema de & Programação & Sob encomenda & 66 \\
\hline & & Diariamente & 9 \\
\hline & & A cada três dias & 16 \\
\hline & Tempo de & Semanalmente & 47 \\
\hline & & Quinzenalmente & 16 \\
\hline & & Mensalmente & 12 \\
\hline & Estoque de peças & Sim & 70 \\
\hline & semiacabadas & Não & 30 \\
\hline & Até $1 \mathrm{r}$ & nilhão & 39 \\
\hline $\begin{array}{l}\text { médio nos } \\
\text { últimos }\end{array}$ & $\begin{array}{r}\text { Acima de } R \$ \\
R \$ 10 n\end{array}$ & $\begin{array}{l}1 \text { milhão a } \\
\text { nilhões }\end{array}$ & 56 \\
\hline 12 meses & Acima de $\mathrm{R} \$$ & 10 milhões & 5 \\
\hline & Não exp & prtamos & 16 \\
\hline Vendas para o & De R\$ $100 \mathrm{mil}$ & a $\mathrm{R} \$ 500 \mathrm{mil}$ & 29 \\
\hline nos últimos & Acima de $R \$ 500 r$ & nil a R\$ 3 milhões & 37 \\
\hline 12 meses & $\begin{array}{r}\text { Acima de } R \$ \\
R \$ 10 \eta\end{array}$ & $\begin{array}{l}3 \text { milhões a } \\
\text { hilhões }\end{array}$ & 18 \\
\hline
\end{tabular}

e Controle de Produção (DPCP); i) Desempenho de Recursos Humanos (DRH); j) Desempenho de Desenvolvimento de Produtos (DDP); k) Desempenho em Qualidade e Custo (DQC); 1) Desempenho em Entrega (IDE); e m) Desempenho em Produtividade (IDP).

Os resultados da AFE foram refinados por uma Análise Fatorial Confirmatória (Confirmatory Factor Analysis - CFA) antes do teste do modelo estrutural (KLINE, 2005). Um método de estimação considerado adequado para dados ordinais é o método dos mínimos quadrados ponderados (Weighted Least Squares - WLS). Kaplan (2000) apresenta uma regra que estabelece um limite de número de variáveis para o tamanho da amostra: se $n$ representa o número de firmas e $p$ o número de variáveis, então,

$\mathrm{p}<\frac{-3+\sqrt{9+8 n}}{2}$

Portanto, no presente trabalho, o modelo estrutural completo não pode ser ajustado com todas as variáveis, conduzindo para especificação de modelos menos complexos, conforme apresentado por Kaplan (2000).

Nesse sentido, a hipótese $\mathrm{H} 1$ se subdividiu em cinco (de H1.1 a H1.5), em que o construto Prioridade Competitiva de Flexibilidade (PCF) foi relacionado com cada um dos cinco construtos de práticas de produção. Assim, a H1 foi reformulada, tal como:

- H1.1 Prioridade competitiva de flexibilidade afeta positivamente práticas de tecnologia para móveis de painéis; e assim sucessivamente para os outros cinco construtos de práticas. A Figura 2 ilustra o modelo estrutural da relação entre o construto de Prioridade Competitiva de Flexibilidade (PCF) e Práticas de Desenvolvimento de Novos Produtos e Mix (PDNPM), onde: $\delta$ representa o erro para as variáveis mensuradas; $\gamma$ representa o coeficiente da carga padronizada para a variável latente; $\xi 0$ construto exógeno; $\eta$ o construto endógeno; $\beta$ o coeficiente estrutural denotando o impacto da prioridade competitiva sobre as práticas operacionais; t a significância estatística do coeficiente $\beta$ (Figura 2); sendo a equação base representada pelos modelos estruturais gerados como:

$\eta=\gamma^{\xi}+\beta \eta+\delta$

A hipótese $\mathrm{H} 2$ se subdividiu em trinta e cinco, em que cada um dos cinco construtos de práticas de produção foi relacionado com cada um dos sete construtos de desempenho de produção. A H2 também foi reformulada, tal como:

- H2.1 Práticas de tecnologia para móveis de painéis afetam positivamente o desempenho de volume de inventário; e assim sucessivamente para os demais construtos de práticas e desempenho. A Figura 3 
apresenta o modelo estrutural da relação entre Práticas de Desenvolvimento de Novos Produtos e de Mix (PDNPM) e desempenho em Planejamento e Controle de Produção (DPCP). A notação da equação é a mesma representada pela Figura 2, sendo, nesse caso, o $\beta$ representando o coeficiente estrutural do impacto das práticas operacionais sobre o desempenho de produção.

Ao todo foram avaliados quarenta modelos de mensuração. Os modelos de mensuração são submodelos das equações estruturais, descrevem o relacionamento entre fatores latentes e suas variáveis indicadoras (HAIR JUNIOR et al., 2005a). Os modelos de mensuração proveem um bom ajuste dos dados e por isso antecedem os modelos de equações estruturais (HATCHER, 1994).

As análises dos resultados gerados dos modelos de mensuração tiveram início a partir da avaliação de estimativas transgressoras. Os exemplos mais comuns de estimativas transgressoras são: a) variâncias dos erros negativas ou não significativas; b) coeficientes padronizados excedentes ou muito próximo de 1,0 ; b) erros padrão muito grandes associados aos coeficientes estimados (HAIR JUNIOR et al., 2005a).

Posteriormente também foram observados os valores $t$, os erros padrão, os resíduos padronizados, as estimativas transgressoras das cargas padronizadas das variáveis, o percentual das variâncias das variáveis endógenas, que é explicado pelo modelo proposto $\left(\mathrm{R}^{2}\right)$ e as estatísticas de qualidade de ajuste. Dessa forma, no decorrer das análises, algumas variáveis foram excluídas, cujos resultados não atenderam aos limites aceitáveis recomendados pela literatura (BOLLEN, 1989; KAPLAN, 2000; HAIR JUN1OR et al., 2005a; KLINE, 2005) e novamente os modelos de mensuração foram avaliados.

Todos os resultados dos cinco modelos da hipótese $\mathrm{H} 1$ foram satisfatórios. Por outro lado, vinte e quatro modelos da hipótese $\mathrm{H} 2$ foram rejeitados, pois apresentaram índices de qualidade de ajuste inaceitáveis. Em outras palavras, tais modelos apresentaram a raiz do resíduo quadrático médio (Root Mean Square Residual, RMR) e a raiz do erro quadrático médio de aproximação (Root Mean Square Error of Approximation, RMSEA) acima de 0,10, indicando um ajuste ruim (KLINE, 2005). Além disso, alguns modelos apresentaram o nível de significância (p-value) do quiquadrado $\left(\chi^{2}\right)$ menor que 0,05 , sendo este o valor mínimo aceitável (HAIR JUNIOR et al., 2005a). Assim, foram gerados dezesseis modelos de mensuração satisfatórios (Tabela 1). A Tabela 2 apresenta os índices de qualidade de ajuste.

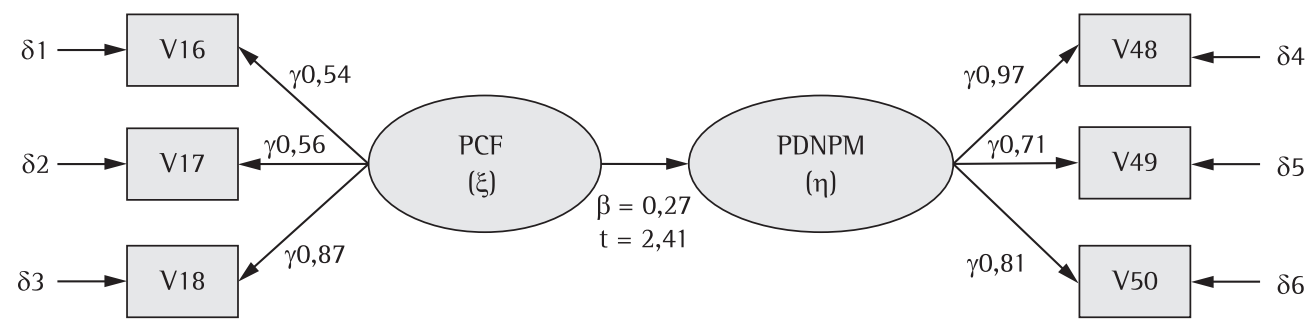

Índices de ajuste:

$\chi 2=12,66 \mathrm{gl}=8 \quad \mathrm{p}$-value $=0,12 \quad \mathrm{GFl}=1,00 \quad \mathrm{RMR}=0,08 \quad \mathrm{NFl}=0,97 \quad \mathrm{AGFl}=1,00 \quad \mathrm{CFl}=0,99 \quad \chi 2 / \mathrm{gl}=1,58$

Figura 2. Resultado do modelo estrutural da relação entre prioridade competitiva de flexibilidade e práticas de desenvolvimento de novos produtos e mix.

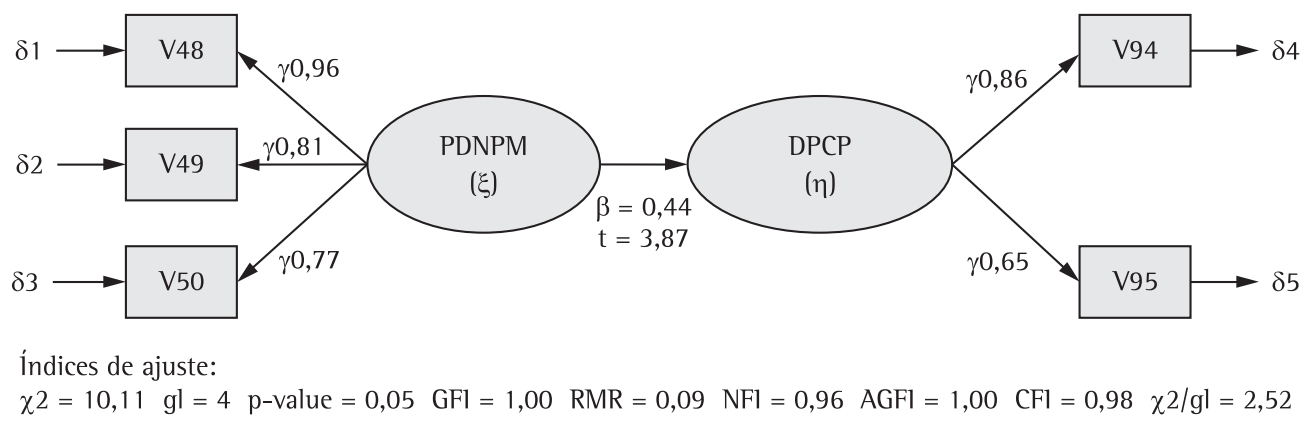

Figura 3. Resultado do modelo estrutural da relação entre práticas de desenvolvimento de novos produtos e mix e desempenho em planejamento e controle de produção. 
Tabela 1. Modelos de mensuração satisfatórios, construtos das hipóteses, confiabilidade e variância extraída.

\begin{tabular}{|c|c|c|c|c|c|c|c|}
\hline $\begin{array}{l}\frac{\tilde{O}}{ \pm} \\
\frac{0}{0} \\
\sum\end{array}$ & $\frac{\sqrt[n]{\pi}}{\frac{\pi}{\pi}}$ & 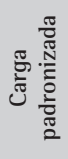 & $\begin{array}{l}\frac{0}{2 \pi} \\
\frac{1}{0} \\
\frac{\pi}{2} \\
\frac{1}{2} \\
\frac{1}{1}\end{array}$ & + & $\approx$ & $\begin{array}{l}\frac{0}{0} \\
\frac{\pi}{0} \\
\vdots 0 \\
\frac{\pi}{0} \\
\frac{\pi}{0} \\
0 \\
0\end{array}$ & 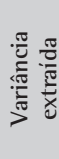 \\
\hline \multirow{8}{*}{ 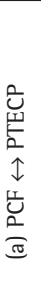 } & Prioridade Competitiva de Flexibilidade (PCF): & - & - & - & - & $0,67^{\mathrm{a}}$ & $60 \%$ \\
\hline & V16-Ter reconhecimento de marca de produto & 0,60 & 0,20 & 3,47 & 0,37 & 0,59 & - \\
\hline & V17-Ter produtos com qualidade design e acabamento & 0,47 & 0,16 & 3,41 & 0,23 & 0,66 & - \\
\hline & V18-Ser a primeira a introduzir novos produtos & 0,85 & - & - & 0,72 & 0,42 & - \\
\hline & Práticas de Tecnologia para Móveis de Painéis (PTECP): & - & - & - & - & $0,72^{\mathrm{a}}$ & $64 \%$ \\
\hline & V23-Máquinas CNC (centro de usinagem, seccionadora) & 0,54 & 0,18 & 3,76 & 0,29 & 0,72 & - \\
\hline & V24-Linha de Pintura UV ou linha de revestimento & 0,67 & 0,18 & 4,58 & 0,45 & 0,62 & - \\
\hline & V25-Máquinas CLP (coladeira de bordo) & 0,81 & - & - & 0,65 & 0,55 & - \\
\hline \multirow{7}{*}{ 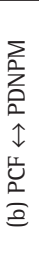 } & V16-Ter reconhecimento de marca de produto & 0,54 & 0,16 & 3,89 & 0,30 & 0,59 & - \\
\hline & V17-Ter produtos com qualidade design e acabamento & 0,56 & 0,16 & 3,93 & 0,32 & 0,66 & - \\
\hline & V18-Ser a primeira a introduzir novos produtos & 0,87 & - & - & 0,76 & 0,42 & - \\
\hline & Práticas de Desenvolvimento de Novos Produtos e de Mix (PDNPM): & - & - & - & - & $0,80^{\mathrm{a}}$ & $72 \%$ \\
\hline & V48-Equipe multifuncional & 0,97 & 0,11 & 10,57 & 0,94 & 0,59 & - \\
\hline & V49-Pesquisa de mercado & 0,71 & 0,09 & 9,71 & 0,50 & 0,85 & - \\
\hline & V50-Documento formal das fases de DNP & 0,81 & - & - & 0,66 & 0,72 & - \\
\hline \multirow{7}{*}{ 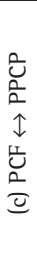 } & V16-Ter reconhecimento de marca de produto & 0,65 & 0,15 & 4,59 & 0,42 & 0,59 & - \\
\hline & V17-Ter produtos com qualidade design e acabamento & 0,51 & 0,13 & 3,92 & 0,26 & 0,66 & - \\
\hline & V18-Ser a primeira a introduzir novos produtos & 0,93 & - & - & 0,86 & 0,42 & - \\
\hline & Práticas de Planejamento e Controle de Produção (PPCP): & - & - & - & - & $0,74^{\mathrm{a}}$ & $66 \%$ \\
\hline & V83-Análise de setup das máquinas & 0,82 & - & - & 0,67 & 0,64 & - \\
\hline & V85-Sistema MRP 11 & 0,68 & 0,13 & 6,20 & 0,46 & 0,67 & - \\
\hline & V88-Roteiros de produção para famílias de produto & 0,74 & 0,12 & 7,38 & 0,55 & 0,68 & - \\
\hline \multirow{8}{*}{ 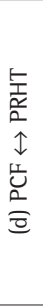 } & V16-Ter reconhecimento de marca de produto & 0,60 & 0,16 & 4,60 & 0,36 & 0,59 & - \\
\hline & V17-Ter produtos com qualidade design e acabamento & 0,56 & 0,16 & 4,20 & 0,31 & 0,66 & - \\
\hline & V18-Ser a primeira a introduzir novos produtos & 0,82 & 0,13 & 8,27 & 0,67 & 0,42 & - \\
\hline & Práticas de Recursos Humanos Tradicionais (PRHT): & - & - & - & - & $0,76^{\mathrm{a}}$ & $59 \%$ \\
\hline & V56-Comprometimento da alta administração & 0,73 & - & - & 0,54 & 0,70 & - \\
\hline & V57-Treinamento formal de funcionários & 0,63 & 0,12 & 7,37 & 0,39 & 0,73 & - \\
\hline & V58-Práticas de incentivos (biblioteca, computadores) & 0,70 & - & - & 0,48 & 0,68 & - \\
\hline & V59-Auxílio para especialização de cursos externos & 0,67 & 0,11 & 8,61 & 0,45 & 0,71 & - \\
\hline \multirow{8}{*}{ 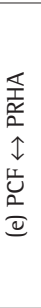 } & V16-Ter reconhecimento de marca de produto & 0,61 & 0,13 & 5,34 & 0,37 & 0,59 & - \\
\hline & V17-Ter produtos com qualidade design e acabamento & 0,60 & 0,13 & 5,28 & 0,36 & 0,66 & - \\
\hline & V18-Ser a primeira a introduzir novos produtos & 0,87 & - & - & 0,75 & 0,42 & - \\
\hline & Práticas de Recursos Humanos Avançadas (PRHA) & - & - & - & - & $0,80^{\mathrm{a}}$ & $63 \%$ \\
\hline & V61-Times de trabalho ou grupos multifuncionais & 0,85 & - & - & 0,72 & 0,76 & - \\
\hline & V62-Sistema de recompensas baseado em habilidades & 0,67 & 0,09 & 8,30 & 0,45 & 0,78 & - \\
\hline & V63-Sistema de recompensas baseado em resultados & 0,77 & 0,09 & 9,22 & 0,60 & 0,75 & - \\
\hline & V64-Sistema de avaliação de desempenho & 0,77 & 0,09 & 9,22 & 0,60 & 0,71 & - \\
\hline \multirow{6}{*}{ 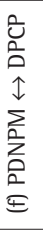 } & V48-Equipe multifuncional & 0,96 & - & - & 0,92 & 0,59 & - \\
\hline & V49-Pesquisa de mercado & 0,81 & 0,10 & 8,10 & 0,66 & 0,85 & - \\
\hline & V50-Documento formal das fases de DNP & 0,77 & 0,08 & 10,43 & 0,60 & 0,72 & - \\
\hline & Desempenho de Planejamento e Controle de Produção (DPCP): & - & - & - & - & $0,71^{\mathrm{a}}$ & $77 \%$ \\
\hline & V94-Flexibilidade para alterar a programação & 0,86 & 0,30 & 4,40 & 0,75 & 0,55 & - \\
\hline & V96-Controle sobre as ordens de produção & 0,65 & - & - & 0,42 & 0,55 & - \\
\hline \multirow{6}{*}{ 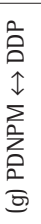 } & V48-Equipe multifuncional & 0,91 & - & - & 0,82 & 0,59 & - \\
\hline & V49-Pesquisa de mercado & 0,69 & 0,11 & 6,75 & 0,47 & 0,85 & - \\
\hline & V50-Documento formal das fases de DNP & 0,74 & 0,10 & 8,30 & 0,55 & 0,72 & - \\
\hline & Desempenho de Desenvolvimento de Produtos (DDP): & - & - & - & - & $0,60^{\mathrm{a}}$ & $71 \%$ \\
\hline & V55-Número de introdução de novos produtos ao mês & 0,71 & 0,37 & 3,27 & 0,51 & 0,42 & - \\
\hline & V67-Número de mudanças no processo produtivo & 0,59 & - & - & 0,35 & 0,42 & - \\
\hline
\end{tabular}

Para os valores $t>1,65: p<0,10 ; t>1,96: p<0,05 ; t>2,56: p<0,01$. ${ }^{a}$ Representa a confiabilidade composta. Os campos sem preenchimento da confiabilidade composta e da variância extraída significam que os construtos se repetem e tais resultados já foram apresentados anteriormente nesta tabela. 
Tabela 1. Continuação...

\begin{tabular}{|c|c|c|c|c|c|c|c|}
\hline $\begin{array}{l}\frac{0}{0} \\
\frac{0}{0} \\
\sum\end{array}$ & 离 & 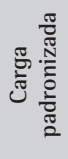 & 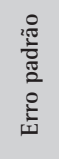 & - & $\approx$ & $\begin{array}{l}\frac{0}{0} \\
\frac{\pi}{0} \\
\frac{0}{0} \\
\frac{\pi}{0} \\
0 \\
0 \\
0\end{array}$ & 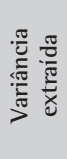 \\
\hline \multirow{7}{*}{ 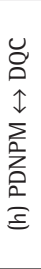 } & V48-Equipe multifuncional & 0,92 & - & - & 0,84 & 0,59 & - \\
\hline & V49-Pesquisa de mercado & 0,69 & 0,10 & 7,84 & 0,48 & 0,85 & - \\
\hline & V50-Documento formal das fases de DNP & 0,82 & 0,10 & 8,85 & 0,68 & 0,72 & - \\
\hline & Desempenho em Qualidade e Custo (DQC): & - & - & - & - & $0,74^{\mathrm{a}}$ & $66 \%$ \\
\hline & V109-índice de retrabalho & 0,72 & - & - & 0,52 & 0,63 & - \\
\hline & V110-Índice de refugo & 0,84 & 0,29 & 4,07 & 0,70 & 0,53 & - \\
\hline & V113-Setup & 0,50 & 0,15 & 4,50 & 0,25 & 0,78 & - \\
\hline \multirow{6}{*}{ 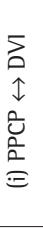 } & V83-Análise de setup das máquinas & 0,76 & - & - & 0,58 & 0,64 & - \\
\hline & V85-Sistema MRP 11 & 0,66 & 0,18 & 4,81 & 0,44 & 0,67 & - \\
\hline & V88-Roteiros de produção para famílias de produto & 0,67 & 0,15 & 5,81 & 0,45 & 0,68 & - \\
\hline & Desempenho de Volume de Inventário (DVI): & - & - & - & - & $0,60^{\mathrm{a}}$ & $71 \%$ \\
\hline & V28-Estoque de matéria-prima & 0,81 & - & - & 0,66 & 0,43 & - \\
\hline & V29-Estoque do produto acabado & 0,66 & 0,36 & 2,26 & 0,44 & 0,43 & - \\
\hline \multirow{5}{*}{ 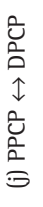 } & V83-Análise de setup das máquinas & 0,74 & - & - & 0,54 & 0,64 & - \\
\hline & V85-Sistema MRP 11 & 0,67 & 0,15 & 6,11 & 0,45 & 0,67 & - \\
\hline & V88-Roteiros de produção para famílias de produto & 0,66 & 0,11 & 7,76 & 0,47 & 0,68 & - \\
\hline & V94-Flexibilidade para alterar a programação & 0,76 & 0,11 & 9,69 & 0,58 & 0,55 & - \\
\hline & V96-Controle sobre as ordens de produção & 0,69 & - & - & 0,48 & 0,55 & - \\
\hline \multirow{6}{*}{ 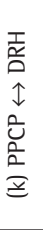 } & V83-Análise de setup das máquinas & 0,80 & - & - & 0,65 & 0,64 & - \\
\hline & V85-Sistema MRP 11 & 0,68 & 0,13 & 6,13 & 0,46 & 0,67 & - \\
\hline & V88-Roteiros de produção para famílias de produto & 0,66 & 0,13 & 6,00 & 0,44 & 0,68 & - \\
\hline & Desempenho de Recursos Humanos (DRH) & - & - & - & - & $0,81^{\mathrm{a}}$ & $84 \%$ \\
\hline & V65-Índice de rotatividade & 0,79 & 0,23 & 3,93 & 0,62 & 0,69 & - \\
\hline & V66-Índice de absenteísmo & 0,84 & - & - & 0,71 & 0,69 & - \\
\hline \multirow{6}{*}{ 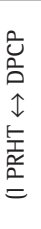 } & V56-Comprometimento da alta administração & 0,76 & 0,16 & 6,74 & 0,57 & 0,70 & - \\
\hline & V57-Treinamento formal de funcionários & 0,54 & 0,15 & 4,97 & 0,29 & 0,73 & - \\
\hline & V58-Práticas de incentivos (biblioteca, computadores) & 0,71 & - & - & 0,51 & 0,68 & - \\
\hline & V59-Auxílio para especialização de cursos externos & 0,59 & 0,15 & 5,52 & 0,35 & 0,71 & - \\
\hline & V94-Flexibilidade para alterar a programação & 0,93 & 0,34 & 4,16 & 0,87 & 0,55 & - \\
\hline & V96-Controle sobre as ordens de produção & 0,65 & - & - & 0,42 & 0,55 & - \\
\hline \multirow{6}{*}{ 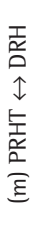 } & V56-Comprometimento da alta administração & 0,82 & 0,17 & 7,18 & 0,67 & 0,70 & - \\
\hline & V57-Treinamento formal de funcionários & 0,51 & 0,15 & 5,09 & 0,26 & 0,73 & - \\
\hline & V58-Práticas de incentivos (biblioteca, computadores) & 0,65 & - & - & 0,43 & 0,68 & - \\
\hline & V59-Auxílio para especialização de cursos externos & 0,62 & 0,17 & 5,59 & 0,39 & 0,71 & - \\
\hline & V65-Índice de rotatividade & 0,73 & - & - & 0,53 & 0,69 & - \\
\hline & V66-Índice de absenteísmo & 0,99 & 0,35 & 3,86 & 0,98 & 0,69 & - \\
\hline \multirow{6}{*}{ 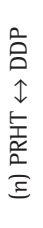 } & V56-Comprometimento da alta administração & 0,69 & 0,12 & 7,34 & 0,48 & 0,70 & - \\
\hline & V57-Treinamento formal de funcionários & 0,70 & 0,11 & 8,23 & 0,49 & 0,73 & - \\
\hline & V58-Práticas de incentivos (biblioteca, computadores) & 0,79 & - & - & 0,63 & 0,68 & - \\
\hline & V59-Auxílio para especialização de cursos externos & 0,58 & 0,10 & 7,21 & 0,34 & 0,71 & - \\
\hline & V55-Número de introdução de novos produtos ao mês & 0,50 & 0,13 & 4,13 & 0,25 & 0,42 & - \\
\hline & V67-Número de mudanças no processo produtivo & 0,96 & - & - & 0,91 & 0,42 & - \\
\hline \multirow{7}{*}{ 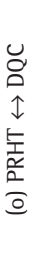 } & V56-Comprometimento da alta administração & 0,67 & 0,13 & 6,52 & 0,45 & 0,70 & - \\
\hline & V57-Treinamento formal de funcionários & 0,61 & 0,12 & 6,34 & 0,37 & 0,73 & - \\
\hline & V58-Práticas de incentivos (biblioteca, computadores) & 0,78 & - & - & 0,60 & 0,68 & - \\
\hline & V59-Auxílio para especialização de cursos externos & 0,76 & 0,14 & 6,88 & 0,59 & 0,71 & - \\
\hline & V109-índice de retrabalho & 0,61 & - & - & 0,37 & 0,63 & - \\
\hline & V110-Índice de refugo & 0,86 & 0,23 & 6,17 & 0,75 & 0,53 & - \\
\hline & V113-Setup & 0,44 & 0,17 & 4,26 & 0,19 & 0,78 & - \\
\hline
\end{tabular}

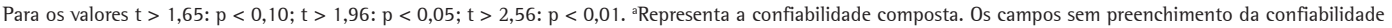
composta e da variância extraída significam que os construtos se repetem e tais resultados já foram apresentados anteriormente nesta tabela. 
Tabela 1. Continuação...

\begin{tabular}{|c|c|c|c|c|c|c|c|}
\hline 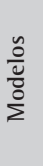 & 迹 & 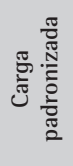 & 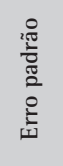 & + & $\approx$ & 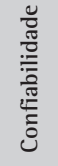 & 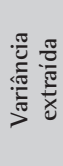 \\
\hline \multirow{6}{*}{ 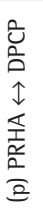 } & V61-Times de trabalho ou grupos multifuncionais & 0,69 & - & - & 0,47 & 0,76 & - \\
\hline & V62-Sistema de recompensas baseado em habilidades & 0,69 & 0,14 & 7,38 & 0,48 & 0,78 & - \\
\hline & V63-Sistema de recompensas baseado em resultados & 0,83 & 0,14 & 8,71 & 0,69 & 0,75 & - \\
\hline & V64-Sistema de avaliação de desempenho & 0,83 & 0,14 & 8,71 & 0,69 & 0,71 & - \\
\hline & V94-Flexibilidade para alterar a programação & 0,89 & 0,30 & 4,43 & 0,79 & 0,55 & - \\
\hline & V96-Controle sobre as ordens de produção & 0,66 & - & - & 0,43 & 0,55 & - \\
\hline
\end{tabular}

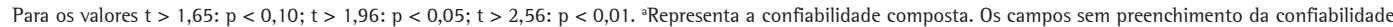
composta e da variância extraída significam que os construtos se repetem e tais resultados já foram apresentados anteriormente nesta tabela.

Tabela 2. Resultados de índices de qualidade de ajuste dos modelos de mensuração.

\begin{tabular}{lrrrrrrrrc}
\hline Modelos de mensuração & \multicolumn{1}{c}{$\chi^{2}$} & gL & Significância & GFI & RMR & NFI & AGFI & CFI & $\chi^{2} /$ gL \\
\hline a) PCF $\leftrightarrow$ PTECP & 4,44 & 8 & 0,81 & 1,00 & 0,04 & 0,96 & 1,00 & 1,00 & 0,55 \\
b) PCF $\leftrightarrow$ PDNPM & 12,66 & 8 & 0,12 & 1,00 & 0,08 & 0,97 & 1,00 & 0,99 & 1,58 \\
c) PCF $\leftrightarrow$ PPCP & 10,59 & 8 & 0,23 & 1,00 & 0,06 & 0,96 & 1,00 & 0,99 & 1,32 \\
d) PCF $\leftrightarrow$ PRHT & 9,44 & 13 & 0,73 & 1,00 & 0,05 & 0,94 & 1,00 & 1,00 & 0,73 \\
e) PCF $\leftrightarrow$ PRHA & 14,73 & 14 & 0,40 & 1,00 & 0,09 & 0,95 & 1,00 & 1,00 & 1,05 \\
f) PDNPM $\leftrightarrow$ DPCP & 10,11 & 4 & 0,05 & 1,00 & 0,09 & 0,96 & 1,00 & 0,98 & 2,52 \\
g) PDNPM $\leftrightarrow$ DDP & 2,97 & 4 & 0,56 & 1,00 & 0,04 & 0,98 & 1,00 & 1,00 & 0,74 \\
h) PDNPM $\leftrightarrow$ DQC & 6,78 & 8 & 0,56 & 1,00 & 0,08 & 0,97 & 1,00 & 1,00 & 0,86 \\
i) PPPCP $\leftrightarrow$ DVI & 3,16 & 4 & 0,53 & 0,99 & 0,03 & 0,96 & 0,99 & 1,00 & 0,79 \\
j) PPCP $\leftrightarrow$ DPCP & 3,66 & 4 & 0,45 & 1,00 & 0,04 & 0,96 & 1,00 & 1,00 & 0,91 \\
k) PPCP $\leftrightarrow$ DRH & 2,46 & 4 & 0,65 & 0,99 & 0,03 & 0,98 & 0,99 & 1,00 & 0,61 \\
l) PRHT $\leftrightarrow$ DPCP & 6,49 & 8 & 0,59 & 1,00 & 0,04 & 0,96 & 1,00 & 1,00 & 0,81 \\
m) PRHT $\leftrightarrow$ DRH & 12,80 & 8 & 0,12 & 1,00 & 0,07 & 0,94 & 1,00 & 0,97 & 1,60 \\
n) PRHT $\leftrightarrow$ DDP & 8,23 & 8 & 0,41 & 1,00 & 0,07 & 0,94 & 1,00 & 1,00 & 1,03 \\
o) PRHT $\leftrightarrow$ DQC & 19,80 & 13 & 0,10 & 1,00 & 0,09 & 0,92 & 1,00 & 0,97 & 1,52 \\
p) PRHA $\leftrightarrow$ DPCP & 14,58 & 9 & 0,10 & 1,00 & 0,09 & 0,95 & 1,00 & 0,98 & 1,62 \\
\hline
\end{tabular}

Para gerar a confiabilidade de cada variável e a confiabilidade composta do construto foi adotado 0 alfa de Cronbach. Os valores variam entre 0 e 1,0. Quanto mais alto, maior é a confiabilidade entre os indicadores (KLINE, 2005). Hair Junior et al. (2005b) consideram baixa confiabilidade coeficientes cujos valores são menores que 0,60 ; confiabilidade moderada, para os valores entre 0,60 e 0,70 ; boa confiabilidade, para os valores maiores que 0,70 e 0,80 ; confiabilidade muito boa, para os valores maiores que 0,80 a 0,90 ; e confiabilidade excelente, para os valores maiores que 0,90 . Conforme Tabela 1 , a confiabilidade composta dos construtos apresentaram valor $\geq 0,60$, indicando confiabilidade moderada, boa e muito boa.

$\mathrm{Na}$ sequência, as validades convergentes e discriminantes foram avaliadas. A validade convergente foi confirmada, uma vez que todas as cargas padronizadas das variáveis apresentaram $t>1,96$ (Tabela 1). Também, Kline (2005) apresenta que a validade convergente é presente quando os indicadores (variáveis) que mensuram um fator em comum possuem carga padronizada relativamente alta sobre tal fator. Observando a Tabela 1, as duas (2) cargas padronizadas mais baixas estão muito próximas de 0,50 (V17:0,47 e V113:0,44) e as demais cargas padronizadas ficaram acima de 0,50, que é um valor considerado alto de acordo com Kline (2005). A validade discriminante foi obtida pelo teste de diferença entre os valores da estatística quiquadrado (HATCHER, 1994; Ll et al., 2005). Entre os dezesseis modelos, um não apresentou a diferença do quiquadrado significativa e foi excluído das análises.

Assim, restaram quinze modelos estruturais. Ao analisar esses modelos, duas hipóteses foram refutadas em razão dos valores $t$ não serem significativos $\mathrm{e}$ dezesseis foram confirmadas (Tabela 3).

Neste ponto, vale apresentar uma discussão sobre a magnitude da carga padronizada que representa o coeficiente estrutural $(\beta)$ da análise de caminhos entre os construtos dos quinze modelos estruturais 
Tabela 3. Resultados dos modelos estruturais e das hipóteses.

\begin{tabular}{lcccc}
\hline $\begin{array}{c}\text { Modelos } \\
\text { estruturais }\end{array}$ & $\begin{array}{c}\text { Carga } \\
\text { padronizada } \\
(\beta)\end{array}$ & $\begin{array}{c}\text { Erro } \\
\text { padrão }\end{array}$ & $t$ & $\begin{array}{c}\text { Resultados } \\
\text { das } \\
\text { hipóteses }\end{array}$ \\
\hline (a) PCF $\rightarrow$ PTECP & $-0,10$ & 0,13 & $-0,73$ & Rejeitada \\
(b) PCF $\rightarrow$ PDNPM & 0,27 & 0,10 & 2,41 & Confirmada \\
(c) PCF $\rightarrow$ PPCP & 0,21 & 0,10 & 1,85 & Confirmada \\
(d) PCF $\rightarrow$ PRHT & 0,35 & 0,11 & 2,76 & Confirmada \\
(e) PCF $\rightarrow$ PRHA & 0,33 & 0,10 & 3,31 & Confirmada \\
(f) PDNPM $\rightarrow$ DPCP & 0,44 & 0,10 & 3,87 & Confirmada \\
(g) PDNPM $\rightarrow$ DDP & 0,71 & 0,14 & 3,36 & Confirmada \\
(h) PDNPM $\rightarrow$ DQC & 0,12 & 0,08 & 1,12 & Rejeitada \\
(i) PPCP $\rightarrow$ DVI & 0,36 & 0,16 & 2,24 & Confirmada \\
(k) PPCP $\rightarrow$ DRH & 0,46 & 0,15 & 3,30 & Confirmada \\
(l) PRHT $\rightarrow$ DPCP & 0,58 & 0,17 & 3,06 & Confirmada \\
(m) PRHT $\rightarrow$ DRH & 0,44 & 0,16 & 3,07 & Confirmada \\
(n) PRHT $\rightarrow$ DDP & 0,72 & 0,10 & 8,35 & Confirmada \\
(o) PRHT $\rightarrow$ DQC & 0,33 & 0,10 & 2,66 & Confirmada \\
(p) PRHA $\rightarrow$ DPCP & 0,52 & 0,15 & 3,18 & Confirmada \\
\hline
\end{tabular}

0 modelo $\mathrm{j}$ foi excluído em razão do resultado do teste da validade discriminante. Para os valores $t>1,65: p<0,10 ; t>1,96: p<0,05 ; t>2,56: p<0,01$.

(Tabela 3). Kline (2005) apresenta que a interpretação da magnitude de tal carga padronizada é complexa. 0 referido autor argumenta que um efeito alto em uma área de pesquisa pode ser considerado um efeito moderado em outra área. Ressaltando ainda que algumas variáveis endógenas são mais difíceis de prever que outras. Dadas essas limitações, Kline (2005) sugere a seguinte interpretação sobre magnitude desta carga padronizada para a área de ciências sociais: um valor menor que 0,10 indica um efeito baixo entre os construtos; valores em torno de 0,30 são considerados um efeito típico ou médio; e os valores maiores ou iguais a 0,50 são considerados efeitos altos. A seção seguinte apresenta uma discussão dos resultados dos testes estatísticos (Tabela 1) e das hipóteses propostas (Tabela 3).

\section{Discussões}

0 construto Prioridade Competitiva de Flexibilidade (PCF) foi, assim, representado pelas variáveis V16 (marca de produto), V17 (design e acabamento) e V18 (introdução de novos produtos) (Tabela 1). Vale ressaltar que apesar da variável marca de produto (V16) ser considerada uma das características de qualidade percebida pelos consumidores, conforme apresentada por Garvin (1988), para as empresas pesquisadas, tal variável estava associada também à introdução de novos produtos (V18). Em modificações contínuas no mix de produtos para móveis planejados retos e também em períodos de lançamento de novas linhas de produtos, a marca da empresa (V16) sofria maior divulgação nos meios de comunicação (catálogos, redes de franquia e lojistas especializados), sendo, portanto, considerada como uma variável que pode representar o construto de Prioridade Competitiva de Flexibilidade.

Ademais, o design e acabamento (V17) também foram associados à marca de produto (V16), pois um design específico e um acabamento do tipo fosco e cores claras, por exemplo, estavam relacionados com uma determinada empresa. Adicionalmente, o construto PCF complementa o estudo de Vickery, Droge e Markland (1997) por inserir as variáveis marca de produto e design e acabamento. No entanto, optou-se por nomeá-lo de flexibilidade, pois a inovação é entendida como uma desagregação da PCF, conforme Garvin (1993).

A PCF afetou positiva e significativamente as práticas de Desenvolvimento de Novos Produtos e de Mix (PDNPM), de Planejamento e Controle de Produção (PPCP), de Recursos Humanos Tradicionais (PRHT) e Avançadas (PRHA), conforme modelos (b), (c), (d) e (e) da Tabela 3, em um efeito médio, conforme cargas padronizadas $(0,27 ; 0,21 ; 0,35$ e 0,33$)$. No entanto, não foi possível atestar que a PCF influencia positivamente as Práticas de Tecnologia de Móveis de Painéis (PTECP), pois o $t$ não foi significativo (modelo a, Tabela 3).

Foi observado que as variáveis que representam o construto PTECP (Tabela 1) se referiam a firmas que manufaturavam móveis de painéis de madeira reconstituída. Assim, é esperado que a associação entre os construtos PCF e PTECP não seja significativa no presente trabalho, uma vez que foi usada a amostra completa de firmas, a qual continha também empresas fabricantes de móveis de madeira maciça, cujas tecnologias eram voltadas para automação fixa. 0 estudo de Kotha e Swamidass (2000) revelou que variáveis como sistemas de controles programáveis baseados em robótica, CAD/CAM, sistemas flexíveis de manufatura e máquinas CNC estavam associadas à flexibilidade e ao baixo volume de produção. Por outro lado, a presente pesquisa mostrou que os investimentos em práticas de tecnologias voltadas para painéis objetivavam a alta automação e o alto volume. Embora os investimentos em máquinas CNC como centro de usinagem, furadeiras e seccionadoras possuíssem potencial de flexibilidade para diferentes programações, as firmas pesquisadas raramente utilizavam esse potencial em razão de estarem preocupadas com a padronização de partes dos produtos.

A relação positiva entre PCF e PDNPM (modelo b, Tabela 3) se justifica pelo sistema de desenvolvimento de novos produtos e de mix adotado nas empresas pesquisadas, que se diferenciava frequentemente conforme o mercado consumidor. Os produtos de madeira maciça, destinados ao mercado externo, tinham 
como clientes grandes lojistas (por ex.: Camif - França; Rooms to Go - EUA) que definiam o design dos móveis, enviando por uma agência comercializante um esboço do produto. 0 protótipo era desenvolvido por uma equipe multifuncional (V48), formada por gerente/ engenheiro de produção, desenhista/projetista, diretor e um operador de fábrica. Nesse processo definia-se o custo do produto, envolvendo entre outros critérios o cálculo da matéria-prima, o processo produtivo e tempo de produção mediante documentação formal (V50). Em seguida, o protótipo era encaminhado ao cliente com a finalidade de negociação.

Para os produtos destinados ao mercado nacional, os clientes se subdividiam em lojistas especializados em móveis (grandes lojistas, como Tok Stok e Etna ou lojas de móveis para bebês), em lojas de marca própria (rede de franquias) e magazines. No mercado nacional o desenvolvimento de produtos ocorria pela própria fábrica (equipe multifuncional) mediante conhecimento prévio de pesquisa de mercado (V49) ou por produtos customizados pelo consumidor final em redes de franquias. Acredita-se que a relação positiva e significativa entre PCF e PDNPM deve-se a essas características.

Nota-se que as Práticas de Planejamento e Controle de Produção (PPCP), mensuradas pelas variáveis V83, V85 e V88 (Tabela 1, modelo c), foram influenciadas positivamente pela PCF (modelo c, Tabela 3) em razão de serem analisados os tempos de setup (V83), sequenciamentos de produção pelo sistema MRP (V85) e roteiro de produção para família de peças (V88) na programação de produção referente à introdução de novos produtos (V18). As observações de um dos autores no chão de fábrica mostraram, por exemplo, que a variável introdução de novos produtos (V18) afetava somente o processo de acabamento (modificações no processo de pintura). Os planos de furos, das furadeiras, ou de perfis, das fresas, não eram alterados. Assim, as alterações de design e acabamento (V17) adotados tiveram influência positiva na análise do setup (V83), pois a atenção em alterações de produtos centrava-se mais no acabamento. As paradas das máquinas para troca de cores de produtos eram menores em quantidade que o setup de furadeiras, fresas e seccionadoras, uma vez que uma mesma cor seria usada em várias linhas de produtos. Assim, eram também estudados os sequenciamentos de produção pelo sistema MRP (V85) e os roteiros de produção para famílias de peças (V88).

Acredita-se que as Práticas de Recursos Humanos Tradicionais (PRHT) e Práticas de Recursos Humanos Avançadas (PRHA) foram influenciadas positiva e significativamente pela PCF (modelos d e e, Tabela 3) como práticas de suporte à implantação bem-sucedida das demais práticas. Os grupos multifuncionais e grupos de melhoria (V61) apoiavam as Práticas de PDNPM e as PPCP mediante a análise do setup das máquinas (V83) e redução de matéria-prima. Por exemplo, no setor de seccionadora, frequentemente os funcionários (ou grupos de melhoria) estudavam um melhor plano de corte de painéis, conforme a inserção de novos produtos, sugerindo melhorias para o programa de corte executado pela seccionadora CNC. Então, eram aproveitados o tempo de setup e o material utilizado nesse setor.

Em se tratando da influência das práticas de produção sobre os indicadores de desempenho $(\mathrm{H} 2)$, foram confirmadas dez relações positivas e significativas entre esses dois construtos (modelos f, g, h, i, q, l, m, n, o e p, Tabela 3).

As PDNPM afetaram positiva e significativamente os Desempenho de Planejamento e Controle de Produção (DPCP) e o Desempenho de Desenvolvimento de Produtos (DDP) (modelo f e modelo g, Tabela 3), considerando a magnitude da carga padronizada de média para alta $(0,44$ e 0,71 , respectivamente). Esse resultado reforça e complementa o trabalho de Tan et al. (2007) por incluir as variáveis V49 (pesquisa de mercado) e V48 (equipes multifuncionais) nessa análise (modelo $f$, Tabela 1). Os referidos autores afirmaram que a habilidade das firmas para responder de forma rápida ao mercado de atuação por meio de práticas de desenvolvimento de novos produtos provê vantagem competitiva. 0 conhecimento das expectativas dos consumidores, mediante a realização de pesquisa de mercado (V49) e, também, do entendimento das necessidades do processo de fabricação, por meio da adoção de equipes multifuncionais (V48), provavelmente melhorou a flexibilidade para alterar a programação de produção (V94) e o controle sobre as ordens de produção (V96). Essas práticas também estimularam a redução de tempo de desenvolvimento de novos produtos afetando maior número de introdução de novos produtos no mês (V55) e o aumento do número de mudanças no processo produtivo por ideias de funcionários (V67), justificando o efeito alto (0,71; Tabela 3 , modelo g) da associação entre as práticas de desenvolvimentos de novos produtos e o desempenho desta área. No entanto, não foi identificada, em observações in loco e nas entrevistas pessoais, alguma relação positiva entre as PDNM com o Desempenho de Qualidade e de Custo, confirmando a rejeição da hipótese, conforme modelo $\mathrm{h}$ (Tabela 3), cuja relação não foi significativa na amostra pesquisada $(\mathrm{t}=1,12)$.

As PPCP afetaram positiva e significativamente o Desempenho de Volume de lnventários (DVI) e o Desempenho de Recursos Humanos (DRH) (modelo i e modelo k, Tabela 3) com um efeito médio entre as associações de tais construtos (0,36 e 0,46, 
respectivamente). Sabe-se que a implantação de sistema MRP 11 (variável V85, modelo i, Tabela 1), que inclui o planejamento agregado de produção, busca obter um melhor planejamento das ordens de produção por meio de previsão de vendas e das necessidades de materiais e como consequência, há uma redução dos níveis de estoque tanto de produto acabado (V28) quanto de matéria-prima (V29). Partindo para a influência de tais práticas sobre o DRH, os índices de rotatividade (V65) e de absenteísmo (V66) (modelo k, Tabela 1) podem ser reduzidos quando há um apoio de práticas de gestão de recursos humanos (Human Resource Management, HRM), conforme identificado por Boselie, Paauwe e Jansen (2001). É possivel que as PPCP implantadas tenham contribuído para a melhoria do DRH, uma vez que houve adoção de Práticas de Recursos Humanos Tradicionais e Avançadas (PRHT e PRHA).

Ambas as PRHT e PRHA afetaram positiva e significativamente o Desempenho de Planejamento de Controle de Produção (DPCP) (modelo 1 e modelo $p$, Tabela 3), com um efeito alto das cargas padronizadas entre as associações $(0,58$ e 0,52 , respectivamente). As PRHT também afetaram positiva e significativamente os Desempenhos de Recursos Humanos (DRH), de Desenvolvimento de Produtos (DDP) e de Qualidade e Custo (DQC) (modelos m, n e o, Tabela 3, respectivamente), com efeitos das cargas padronizadas considerados médio e alto $(0,44,0,72$ e 0,33 , respectivamente). Conforme mencionado anteriormente, é possivel que as PRHT e PRHA tenham influenciado os indicadores de desempenho de outras áreas, visto que essas práticas são consideradas como apoiadoras para as melhores práticas a serem implantadas, corroborando a afirmação de Davies e Kochhar (2000, 2002). Observações no chão de fábrica no presente estudo revelaram que o treinamento formal (V57) e o comprometimento da alta administração para com os funcionários (V56) intensificavam o controle sobre as ordens de produção (V96), a flexibilidade para alterar a programação (V94) e o número de ideias para mudanças no processo produtivo (V67), o que pode justificar a alto efeito (0,72; Tabela 3 , modelo $n)$ das práticas de recursos humanos tradicionais sobre 0 desempenho de desenvolvimento de novos produtos.

Por fim, todas essas análises respondem ao problema de pesquisa do presente trabalho, revelando que as PDNPM, PPCP, PRHT e PRHA podem ser consideradas as melhores práticas para o contexto do setor estudado. Tais práticas estavam alinhadas à estratégia de produção (representadas pelas prioridades competitivas) e afetaram a melhoria de indicadores de desempenho, sendo ambas as relações positivas e com significância estatística. Tal afirmação é corroborada pela revisão teórica, apresentada na Seção 2 do presente trabalho.

\section{Considerações finais, sugestões para trabalhos futuros e limitações da pesquisa}

Este trabalho analisou a influência da estratégia de produção sobre investimentos em práticas e, também, o impacto das práticas sobre a melhoria do desempenho operacional. Mediante essa análise foi possível saber quais eram as melhores práticas de produção no setor pesquisado. Assim, as principais contribuições deste estudo foram: a) fornecer maior esclarecimento das lacunas encontras em estudos prévios, principalmente, referentes à definição de melhores práticas para um contexto específico; b) desenvolver novos construtos sobre práticas de produção e de desempenho operacional, além de variáveis específicas para o setor moveleiro. As variáveis que compuseram cada construto não são exatamente as mesmas utilizadas por outros estudos, pois foram inseridas novas variáveis específicas para o setor moveleiro e outras foram eliminadas no decorrer do refinamento da validação dos construtos. Assim, tendo em vista o rigor metodológico empregado, estudos subsequentes poderão usar os mesmos construtos e investigar novos relacionamentos, envolvendo outros construtos de práticas de produção e de indicadores de desempenho; c) relacionar práticas de áreas específicas com indicadores de desempenho de diferentes áreas de produção, confirmando que as práticas podem afetar outras áreas da organização; e d) adotar um método estatístico ainda pouco empregado na área de gerenciamento de operações - a revisão da literatura apontou que poucos estudos utilizaram a técnica de SEM com o método de estimação WLS. Essa metodologia é particularmente adequada para dados ordinais (escalas tipo Likert), como empregado neste trabalho.

No âmbito gerencial das organizações, o presente trabalho promoveu um esclarecimento sobre a adoção dessas práticas, encorajando gerentes a realizar um planejamento minucioso acerca de quais práticas adotar e, também, desenvolver e mensurar indicadores continuadamente a fim de aferir os resultados alcançados em médio e longo prazo.

Sugere-se, para futuros estudos, inserir outras variáveis de pesquisa a fim de obter maior validação dos construtos pela técnica estatística. Por exemplo, inserir na área de desenvolvimento de novos produtos as variáveis: a) desenvolvimento modular e; b) padronização de partes de componentes. Notou-se que o setor pesquisado padroniza algumas peças de linhas de produtos para reduzir o setup de furadeiras e de fresas.

Em se tratando das prioridades competitivas, a variável design e acabamento (V17) pode ser dividida em duas variáveis novas, uma vez que ambos os 
critérios (design e acabamento) são considerados distintos, conforme a matéria-prima utilizada.

Os efeitos diretos e indiretos de um dos construtos sobre os outros dois também poderiam ser avaliados pela técnica estatística, se o modelo fosse conduzido por completo (com todas as variáveis do estudo). No presente estudo, não foi possível realizar esta análise em decorrência do número de firmas pesquisadas. Os entrevistados (gerentes de produção) tinham um perfil de atuação de forma direta com o chão de fábrica na maior parte das horas de trabalho, sendo que o uso do computador geralmente ocorria fora de expediente, acarretando uma maior resistência para responder pesquisas pela Internet. Não obstante, muitos esforços foram feitos para obter dados precisos e os resultados dos testes estatísticos foram rigorosamente aferidos, sobretudo, os testes de validade e confiabilidade dos construtos e, também, o desenvolvimento de modelos estruturais menos complexos que foram condizentes com o tamanho da amostra pesquisada, conforme regra apresentado por Kaplan (2000) na Seção 3 deste artigo.

Ressalta-se que o presente trabalho considerou a análise entre estratégia de produção, práticas e desempenho para toda a amostra, sem distinguir características do setor moveleiro, tais como móveis retilíneos ou torneados, ou ainda porte da empresa. Justifica-se que não foi possível fazer distinções das características em razão do tamanho da amostra para o emprego da técnica de modelagem de equações estruturais, pois a distinção entre empresas limitaria o uso de variáveis importantes para o setor estudado. Assim, o presente estudo fez as análises considerando todas as características do setor moveleiro. Apesar dessa limitação, acredita-se que a distinção entre características não afetariam os resultados obtidos, pois as práticas utilizadas e avaliadas são aplicadas a todas as empresas do setor. Por fim, estudos futuros poderiam empregar tais relações em outros setores.

\section{Referências}

ADAM, E. E.; SWAMIDASS, P. M. Assessing operations management from strategic perspective. Journal of Management, v. 15, n. 2, p. 181-203, 1989. http://dx.doi.org/10.1177/014920638901500204

ANGELL, L. C.; KLASSEN, R. D. Integrating environmental issues into the mainstream: an agenda for research in operations management. Journal of Operations Management, v. 17, n. 5, p. 575-598, 1999. http://dx.doi.org/10.1016/S0272-6963(99)00006-6

BEAMON, B. M. Measuring supply chain performance. International Journal of Operations \& Production Management, v. 19, n. 3-4, p. 275-292, 1999. http://dx.doi.org/10.1108/01443579910249714
BEAUMONT, N. Best practice in Australian manufacturing sites. Technovation, v. 25, n. 11, p. 1291-1297, 2005. http://dx.doi.org/10.1016/j.technovation.2004.09.004

BOLLEN, K. A. Structural equations with latent variables. New York: Wiley, 1989.

BOSELIE, P.; PAAUWE, J.; JANSEN, P. Human resource management and performance: lessons from the Netherlands. International Journal of Human Resource Management, v. 12, n. 7, p. 1107-1125, 2001. http://dx.doi.org/10.1080/09585190110068331

BOYER, K. K. Longitudinal linkages between intended and realized operations strategies. International Journal of Operations \& Production Management, v. $18, \quad$ n. $4, \quad$ p. $356-373,1998$. http://dx.doi. org/10.1108/01443579810199739

CAMP, R. C. Benchmarking: the search for industry best practices that lead to superior performance. Milwaukee: ASQC Quality Press, 1999.

CENTRO GESTOR DE INOVAÇÃO MOVELEIRO - AGIM. Cenário Moveleiro. Disponível em: <http://www. cgimoveis.com.br>. Acesso em: 4 jun. 2007.

CUA, K. O.; McKONE, K. E.; SCHROEDER, R. G. Relationships between implementation of TQM, JIT and TPM and manufacturing performance. Journal of Operations Management, v. 19, n. 6, p. 675-694, 2001. http:// dx.doi.org/10.1016/S0272-6963(01)00066-3

DAVIES, A. J.; KOCHHAR, A. K. A framework for the selection of best practices. International Journal of Operations \& Production Management, v. 20, n. 10, p. 1203-1217, 2000. http://dx.doi.org/10.1108/01443570010343744

DAVIES, A. J.; KOCHHAR, A. K. Manufacturing best practice and performance studies: a critique. International Journal of Operations \& Production Management. International Journal of Operations \& Production Management, v. 22, n. 3, p. 289-305, 2002. http:// dx.doi.org/10.1108/01443570210417597

DíAZ, M. S.; GlL, M. J. A.; MACHUCA, J. A. D. Performance measurement systems, competitive priorities, and advanced manufacturing technology Some evidence from the aeronautical sector. International Journal of Operations \& Production Management, v. 25, n. 8, p. 781-799, 2005. http://dx.doi. org/10.1108/01443570510608600

FINE, C. H.; HAX, A. C. Manufacturing strategy: a methodology and an illustration. Interfaces, v. 15, n. 6 , p. 28-46, 1985. http://dx.doi.org/10.1287/inte.15.6.28

FLYNN, B. B. et al. World-class manufacturing project: overview and selected results. International Journal of Operations \& Production Management, v. 17, n. 7, p. 671-685, 1997. http://dx.doi.org/10.1108/01443579710175592

FORTUIN, L. Performance indicators: why, where and how? European Journal of Operational Research, v. 34, n. 1, p. 1-9, 1988. http://dx.doi.org/10.1016/03772217(88)90449-3

FOWLER, F. J. Survey research methods. Newbury: Sage, 2002.

FREITAS, H. et al. 0 método de pesquisa survey. RAUSPRevista de Administração, v. 35, n. 3, p. 105-112, 2000.

FYNES, B.; VOSS, A. C.; BÚRCA, S. The Impact Of Supply chain relationship dynamics on manufacturing performance. International Journal of Operations \& Production Management, v. 25, n. 1, p. 6-19, 2005. http://dx.doi. org/10.1108/01443570510572213 
GARVIN, D. A. Managing quality: the strategic and competitive edge. New York: The Free Press, 1988.

GARVIN, D. A. Manufacturing strategic planning. California Management Review, v. 35, n. 4, p. 85-106, 1993.

GERWIN, D. A Agenda for research on the flexibility of manufacturing processes. International Journal \& Production Management, v. 7, n. 1, p. 38-49, 1987. http://dx.doi.org/10.1108/eb054784

GILLEY, M. K.; RASHEED, A. Making more by doing less: an analysis of outsourcing and its effects on firm performance. Journal of Management, v. 26, n. 4, p. 763-790, 2000.

HAIR JUNIOR, J. F. et al. Multivariate Data Analysis. Upper Saddle River: Prentice Hall, 2005 a.

HAIR JUNOIR, J. F. et al. Fundamentos de métodos de pesquisa em administração. Porto Alegre: Bookman, 2005b.

HATCHER, L. A step-by-step approach to using SAS for factor analysis and structural equation modeling. Carey: SAS Institute, 1994.

HAYES, R. H. et al. Operations, Strategy and Technology, Pursuing the Competitive Edge. New York: John Willey \& Sons, 2004.

HAYES, R. H.; WHEELWRIGHT, S. C. Restoring our competitive edge: competing through manufacturing. New York: John e Wiley, 1984.

HILL, T. Manufacturing strategy: text and cases. Richard D. Irwin, 1993.

HÖRTE, S. A.; LINDBERG, L.; TUNÄLV, C. Manufacturing strategies in Sweden. International Journal of Production Research, v. 25, n. 11, p. 1573-1586, 1987.

INSTITUTO DE ESTUDOS E MARKETING INDUSTRIAL - IEMI. Relatório Setorial da Indústria de Móveis no Brasil. São Paulo: IEMl, 2006. v. 1.

KAPLAN, D. Structural equation modeling: foundations and extensions. Newbury Park: Sage Publications, 2000.

KETOKIVI, M.; SCHROEDER, R. G. Manufacturing practices, strategic fit and performance: a routine-based view. International Journal of Operations \& Production Management, v. 24, n. 2, p. 171-191, 2004. http:// dx.doi.org/10.1108/01443570410514876

KLINE, R. B. Principles and practice of structural equation modeling. New York: Guilford, 2005.

KOTHA, S.; SWAMIDASS, P. M. Strategy, advanced manufacturing technology and performance: empirical evidence from U.S. manufacturing firms. Journal of Operations Management, v. 18, n. 3, p. 257-277, 2000. http://dx.doi.org/10.1016/S0272-6963(99)00025-X

LAUGEN, T. B.; BOER, N. A. H.; FRICK, J. Best manufacturing practices: what do the best performing companies do? International Journal of Operations \& Production Management, v. 25, n. 2, p. 131-150, 2005. http://dx.doi.org/10.1108/01443570510577001

LEONG, G. K.; SNYDER, D. L.; WARD, P. Research in the process and content of manufacturing strategy. OmegaInternational Journal of Management Science, v. 18, n. 2, p. 109-122, 1990. http://dx.doi.org/10.1016/03050483(90)90058-H

$\mathrm{Ll}$, S. et al. Development and validation of a measurement instrument for studying supply chain management practices. Journal of Operations Management, v. 23, n. 6, p. 618-641, 2005. http://dx.doi.org/10.1016/j. jom.2005.01.002

$\mathrm{Ll}$, S. et al. The impact of supply chain management practices on competitive advantage and organizational performance. Omega-International Journal of Management Science, v. 34, n. 2, p. 107-124, 2006. http://dx.doi.org/10.1016/j. omega.2004.08.002

MILLER, J. G.; ROTH, A. V. A taxonomy of manufacturing strategies. Management Science, v. 40, n. 3, p. 285-304, 1994. http://dx.doi.org/10.1287/mnsc.40.3.285

MILLS, J.; PLATTS, K.; GREGORY, M. A framework for the design of manufacturing strategy process: a contingency approach. International Journal of Operations \& Production Management, v. 15, n. 4, p. 17-49, 1995. http://dx.doi.org/10.1108/01443579510083596

NARASIMHAN, R.; SWINK, M.; KIM, S. W. An exploratory study of manufacturing practiceand performance interrelationships: implications for capability progression. International Journal of Operations \& Production Management, v. 25, n. 10, p. 1013-1033, 2005. http://dx.doi.org/10.1108/01443570510619509

PAIVA, E. L.; CARVALHO JUNIOR; J. M.; FENSTERSEIFER, J. E. Estratégia de produção e de operações: conceitos, melhores práticas e visão de futuro. Porto Alegre: Bookman, 2004.

PINSONNEAULT, A.; KRAEMER, K. L. An assessment of the use of survey research in the management information systems (MIS) field between 1980 and 1990. Journal of Management Information Systems, v. 10, n. 2, p. 75-106, 1993.

PIRES, S. R. 1. Gestão estratégica da produção. Piracicaba: Unimep, 1995.

PUJARI, D. Eco-innovation and new product development: understanding the influences on market performance. Technovation, v. 26, n. 1, p. 76-85, 2006. http://dx.doi org/10.1016/j.technovation.2004.07.006

SCHMENNER, R. W. Production/operations management, concepts and situations. Chicago: Science Research Associates, 1981.

SCHROEDER, R. G. Operations management. New York: McGraw-Hill, 1993.

SCHROEDER, R. G.; BATES, K. A.; JUNTTILA, M. A. A resource-based view of manufacturing strategy and the relationship to manufacturing performance. Strategic Management Journal, v. 23, n. 2, p. 105-117, 2002. http://dx.doi.org/10.1002/smj.213

SEBRAE. Critérios para definição do porte das empresas. Sebrae, 2008. Disponivel em: <http://www.ms.sebrae. com.br/OrientacaoEmpresarial/estudos/>.

SILA, 1. Examining the effects of contextual factors on TQM and performance through the lens of organizational theories: an empirical study. Journal of Operations Management, v. 25, n. 1, p. 83-109, 2007. http://dx.doi. org/10.1016/j.jom.2006.02.003

SILA, 1.; EBRAHIMPOUR, M. Critical linkages among TQM factors and business results. International Journal \& Production Management, v. 25, n. 11, p. 1123-1155, 2005. http://dx.doi.org/10.1108/01443570510626925

SILVA, E. M. et al. Análise da relação entre a dimensão ambiental e as prioridades competitivas tradicionais de produção: um estudo em empresas com certificação ISO 14001. In: ANNUAL MEETING DA ANPAD - ENANPAD, 32., 2008. Anais... Rio de Janeiro: ANPAD, 2008. 
SKINNER, W. Manufacturing: the missing link in corporate strategy. Harvard Business Review, v. 47, n. 3, p. 136-145, 1969.

SKINNER, W. Manufacturing in the corporate strategy. New York: Wiley, 1978.

SOUSA, R.; VOSS, A. C. Contingency research in operations management practices. Journal of Business and Management, v. 26, n. 6, p. 697-713, 2008.

STONENRAKER, P. W.; LEONG, G. K. Operations strategy. Massachusetts: Allyn and Bacon, 1994.

SWAMIDASS, P. M.; NEWELL, W. T. Manufacturing strategy, environmental uncertainty and performance: a path analytic model. Management Science, v. 33, n. 4, p. 509-524, 1987. http://dx.doi.org/10.1287/mnsc.33.4.509

SWINK, M.; NARASIMHAN, R.; WANG, C. Managing beyond the factory walls: Effects of four types of strategic integration on manufacturing plant performance. Journal of Operations Management, v. 25, n. 1, p. 148-164, 2007. http://dx.doi.org/10.1016/j.jom.2006.02.006

SYNODINOS, N. E. The "art" of questionnaire construction: some important considerations for manufacturing studies. Integrated Manufacturing Systems, v. 14, n. 3, p. 221-237, 2003. http://dx.doi. org/10.1108/09576060310463172

UNGAN, M. Management support for the adoption of manufacturing best practices: key factors. International Journal of Operations \& Production Management, v. 43, n. 18, p. 3803-3820, 2005.
VACHON, S.; KLASSEN, R. D. Green project partnership in the supply chain: the case of the package printing industry. Journal of Cleaner Production, v. 14, n. 6-7, p. 661-671, 2006. http://dx.doi.org/10.1016/j. jclepro.2005.07.014

VICKERY, S. K.; DROGE, C.; MARKLAND, R. E. Dimensions of manufacturing: strength in the furniture industry. Journal of Operations Management, v. 15, n. 4, p. 317-330, 1997. http://dx.doi.org/10.1016/S02726963(97)00012-0

VOSS, A. C. Alternative paradigms for manufacturing strategy. International Journal of Operations \& Production Management, v. 15, n. 4, p. 5-16, 1995. http://dx.doi. org/10.1108/01443579510083587

WARD, P. T.; McCREERY, J. K.; RITZMAN, L. P. Competitive priorities in operations management. Decision Sciences, v. 29, n. 4, p. 1035-1046, 1998. http://dx.doi. org/10.1111/j.1540-5915.1998.tb00886.x

WHEELWRIGHT, S. C. Manufacturing strategy: defining the missing link. Strategic Management Journal, v. 5, n. 1, p. 77-91, 1984. http://dx.doi.org/10.1002/ smj.4250050106

WOMACK, J. P.; JONES, D. R.; ROOS, D. The machine that changed the world. New York: Rawson Associates, 1990.

YOUNDT, M. A. et al. Human resource management, manufacturing strategy and firm performance. Academy of Management Journal, v. 39, n. 4, p. 836-866, 1996. http://dx.doi.org/10.2307/256714

\title{
Analysis of the relationship between manufacturing strategy, practices and operational performance
}

\begin{abstract}
The objective of this work was to identify the best production practices for the furniture sector through the analysis of the relationship between implemented production practices, competitive priorities and improvement on operational performance indicators. The empirical research was an explanatory cross-sectional survey. Data were collected from interviews and direct observation on the shop-floor of 99 furniture companies. The technique used for data analysis was the Structural Equation Modeling (SEM) with the Weighted Least Square (WLS) estimation method. Results indicated that practices in the area of development of new products, human resources and production planning and control were aligned with manufacturing strategy and contributed to enhance operational performance indicators, therefore being regarded as best practices in the context under study. Discussion and suggestions for further research have been added.
\end{abstract}

Keywords

Best practices. Manufacturing strategy. Operational performance. Structural equation modeling. Furniture sector. 